\title{
Screening for Melanoma in Primary Care
}

\author{
S Ribero ${ }^{* 1,2}$ and V Bataille ${ }^{1,3}$ \\ ${ }^{1}$ Twin Research and Genetic Epidemiology Unit, Kings College, UK \\ ${ }^{2}$ Medical sciences department, University of Turin, Italy \\ ${ }^{3}$ Dermatology Department, West Herts NHS Trust, UK
}

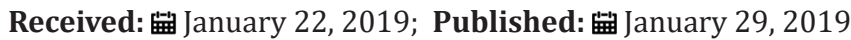

*Corresponding author: Simone Ribero, Department of Twin Research and Genetic Epidemiology, King's College London, St Thomas' Campus, Westminster Bridge Road, London

\begin{abstract}
Melanoma screening is a controversial topic in the literature. Advanced melanoma is an incurable disease with complex and expensive treatments. Melanoma is particularly amenable to secondary prevention as it is visible on the skin and more than $80 \%$ of melanomas will not have any recurrence if detected early. It is therefore important to recognise early melanoma but also to identify patients at future risk with the most predictable phenotypic and genetic risk factors. Neavus count has been described as the main risk factor for melanoma. However, total body skin examinations and mole check are not often performed during GP visits because of time constraints. Naevi typically involute after the fourth decade of life. Individuals with a susceptibility to melanoma often have large numbers of common and atypical naevi which persist until middle age or later. This review focus on the phenotypic risks associated with melanoma as well the relevance of family history.
\end{abstract}

\section{Introduction}

A The incidence of malignant melanoma is increasing worldwide [1]. General Practitioners (GPs) see a large number of skin lesions in their day to day practice and their role in detecting early melanoma is very important. In Europe, a preferential road has been set up to speed up the referral of suspected cancer since almost 20 years [2]. However, many clinics predisposed to screen high risk population see a large number of benign lesions and the ratio of malignant to benign can be very low. It is important to establish the most important risk factors for melanoma by a medical history including family history and examination of the whole body if times allows. Regarding the target lesion, GPs can increase their skills using dermoscopy which if used on a regular basis can significantly increase diagnostic skills.

\section{Text}

GPs often ask about previous sunburns and lifetime sun exposure when assessing skin cancer risk. However, this is not discriminating well between low and high-risk melanoma patients as sun exposure is so ubiquitous. Total body naevus count (TBNC) is one of the most important risk factor for melanoma with much higher relative risks than environmental factor (sun exposure) [3] Naevi are under genetic control and a smaller percentage of the variance in naevus counts may be explained by sun exposure $[4,5]$ Naevi confer the same magnitude of risk for melanoma at all latitudes in Caucasian populations suggesting that sun exposure is not an important factor for this association. Naevi number genetics has helped in discovering new melanoma genes but this phenotype is also informative as it does unrevel some associations between melanoma risk factors and other phenotypes such as reduced ageing and longer telomeres [6-8]. Naevi typically involute after the fourth decade of life. Individuals with a susceptibility to melanoma often have large numbers of common and atypical naevi which persist until middle age or later [9]. Patients presenting with multiple naevi, especially atypical, have a delayed senescence and keep their naevi longer with age. So, assessing naevus counts in individuals age 45 years and over is a very sensitive way to detect patients most at risk of melanoma.

Body sites are also very important when assessing naevi [10]. For example, intradermal naevi are common on the face whilst they are rarer on the limbs for example. Atypical naevi on the contrary, are common on the trunk and proximal limbs but rarer on the face and distal limbs. Knowing the distribution of different types of naevi on the body and their biology helps to determine whether a lesion may be suspicious or not. Naevi are also more common in fair skin individuals and decrease the darker the skin type within Caucasians. In non-Caucasians, multiple naevi and especially atypical naevi are very uncommon and in these patients, other diagnoses such as dermatofibroma, seborrhoeic keratosis, haemangioma or darkly 
pigmented intradermal naevus are more likely when diagnosing a pigmented lesion. Melanoma in children is also extremely rare and yet the high-risk clinic will also encounter children referred for suspected melanoma. The most common diagnosis in children will be benign naevi or haemangiomas. Dermatofibromas and seborrhoeic keratoses are rare in children. Naevi do change with age over years rather than weeks or month and it is common to see lesions becoming bigger as the child grow. Children are also more likely to present with naevi on the torso, face, neck and scalp if the family is quite Moley as naevi are under significant genetic control.

The removal of pigmented lesion in children should only proceed after the child has been examined by an experienced dermatologist who in most instances will be reassuring and will avoid biopsy in children. In clinical practice, naevus count is a very important clinical marker of melanoma risk and easy to document [11]. However, total body skin examinations and mole check are not often performed during GP visits because of time constraints. Many studies have used naevus count on selected body sites in order to identify patients at-risk of melanoma as TBNC is time consuming in general practice [12]. We previously demonstrated that having an arm naevus count of more than 11 is associated with a higher risk of having more than 100 naevi, which is a strong risk factor for melanoma [13] This tool should be used for a quick estimation of melanoma risk in general practice. Moreover, patients would be able to count their moles and ask their GPs to confirm the risk. GPs could then examine the patients and on the basis of a quick arm count (or total body naevus count if times allows), as well as family history of melanoma and other cancers, the GPs could decide which patients are most at risk of melanoma and refer if appropriate. Patients self-examination is reported to be a good practical approach for successful early detection in high risk population for melanoma screening in the USA [14].

Self-examination is rare in the general population that is often not aware of the most important risk factor for melanoma which is naevus number. We are conscious that offering a screening visit to the whole population above the age of 45 years without selection (such as in Germany) [15] will be too time consuming and not feasible in Europe. Furthermore, the pickup rate of melanoma would be too low to be worthwhile. It is therefore important to educate patients that moles disappear with age and if these remain numerous after the age of 50 years, this should be regarded as a significant risk factor for melanoma. The most common message given in public health campaigns for skin cancer and especially melanoma, is that sunshine exposure is the most important risk factor. This does not pick up patients most at risk as it is not discriminatory and fair skin which burns easily is too common in Europe to be a reliable "at risk" phenotype for melanoma. Family history of all types of cancers is also important when assessing melanoma risk as the presence of multiple atypical naevi and increased melanoma risk is more likely to occur in the context of family cancer syndromes. Clustering of different tumours within family at risk is not uncommon and especially for brain, pancreas, breast, colon and kidney cancer.
For many cancers, the health systems provide screening such as mammography, PAP test, PSA and faecal occult blood for example, which can be expensive and time consuming. For melanoma, a simple screening for skin cancer (not invasive, cheap and with limited equipment such as a dermoscope which is not absolutely necessary) there is no agreed national program even from a specified age group. GPs are now recommended to be trained in dermoscopy for the diagnosis of skin tumours in the latest NICE melanoma guidelines [16]. So, the roles of GPs in the diagnosis of early melanoma will increase in the next 10 years. GPs can quickly acquire good diagnostic skills using dermoscopy if they use it routinely in their practice. Basic dermoscopy courses with a lot of practical cases are also helpful. Advanced dermoscopy courses are not recommended for GPs as they may confuse them and be too complex which often lead to GPs being inhibited once back in their practice. By teaching GPs how to select high risk groups for melanoma, it is likely that the yield of melanoma detected in primary care will increase by more appropriate referrals to the 2 weeks wait clinics. It will also sensitise patients about the most important risk factors for melanoma via self-examination.

\section{References}

1. Ribero S, Glass D, Bataille V (2016) Genetic epidemiology of melanoma. Eur J Dermatol 26(4): 335-339.

2. Dodds W, Morgan M, Wolfe C, Raju KS (2004) Implementing the 2-week wait rule for cancer referral in the UK: general practitioners' views and practices. Eur J Cancer Care (Engl) 13(1): 82-87.

3. Garbe C, Büttner P, Weiss J, Soyer HP, Stocker U, et al. (1994) Associated factors in the prevalence of more than 50 common melanocytic naevi, atypical melanocytic naevi, and actinic lentigines: multicenter casecontrol study of the Central Malignant Melanoma Registry of the German Dermatological Society. J Invest Dermatol 102(5): 700-705.

4. Bataille V, Snieder H, MacGregor AJ, Sasieni P, Spector TD (2000) Genetics of risk factors for melanoma: an adult twin study of naevi and freckles. J Natl Cancer Inst 92(6): 457-463.

5. Wachsmuth RC, Turner F, Barrett JH, Gaut R, Randerson Moor JA, et al. (2005) The effect of sun exposure in determining naevus density in UK adolescent twins. J Invest Dermatol 124(1): 56-62.

6. Bataille V, Kato BS, Falchi M, Gardner J, Kimura M, Lens M, et al. (2007) Nevus size and number are associated with telomere length and represent potential markers of a decreased senescence in vivo. Cancer Epidemiol Biomarkers Prev 16(7): 1499-1502.

7. Ribero S, Glass D, Aviv A, Spector TD, Bataille V (2015) Height and bone mineral density are associated with naevus count supporting the importance of growth in melanoma susceptibility. PLoS One 10(1): e0116863.

8. Ribero S, Mangino M, Bataille V (2016) Skin phenotypes can offer some insight about the association between telomere length and cancer susceptibility. Med Hyp 97: 7-10.

9. Newton JA, Bataille V, Griffiths K, Squire JM, Sasieni P, et al. (1993) How common is the atypical naevus syndrome phenotype in apparently sporadic melanoma? J Am Acad Dermatol 29(6): 989-996.

10. Ribero S, Osella Abate S, Reyes Garcia D, Glass D, Bataille V (2017) Gender effects on naevi body distribution and melanoma risk in two melanoma case control studies at different latitudes. Br J Dermatol in press 176(4): 1093-1094.

11. Smith RA, Cokkinides V, Brawley OW (2009) Cancer screening in the United States, 2009: a review of current American Cancer Society guidelines and issues in cancer screening. CA Cancer J Clin 59(1): 27-41. 
12. Echeverría B, Bulliard JL, Guillén C, Nagore E (2014) Indicators for the total number of melanocytic naevi: an adjunct for screening campaigns. Observational study on 292 patients. Br J Dermatol 170(1): 144-149.

13. Ribero S, Zugna D, Osella Abate S, Glass D, Nathan P, et al. (2016) Prediction of high naevus count in a healthy UK population to estimate melanoma risk. Br J Dermatol 174(2):312-318.

14. Swetter SM, Pollitt RA, Johnson TM, Brooks DR, Geller AC (2012) Behavioral determinants of successful early melanoma detection: role of self and physician skin examination. Cancer 118(15): 3725-3734.

\section{ISSN: 2574-1241}

DOI: 10.26717/BJSTR.2019.13.002453

Simone Ribero. Biomed J Sci \& Tech Res

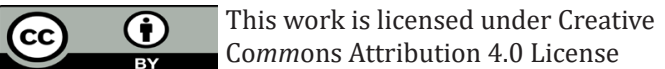

Submission Link: https://biomedres.us/submit-manuscript.php
15. Breitbart EW, Waldmann A, Nolte S, Capellaro M, Greinert R, et al. (2012) Systematic skin cancer screening in Northern Germany. J Am Acad Dermatol 66(2): 201-211.

16. Macbeth F, Newton Bishop J, O’Connell S, Hawkins JE (2015) Melanoma: summary of NICE guidance. BMJ 351: h3708.

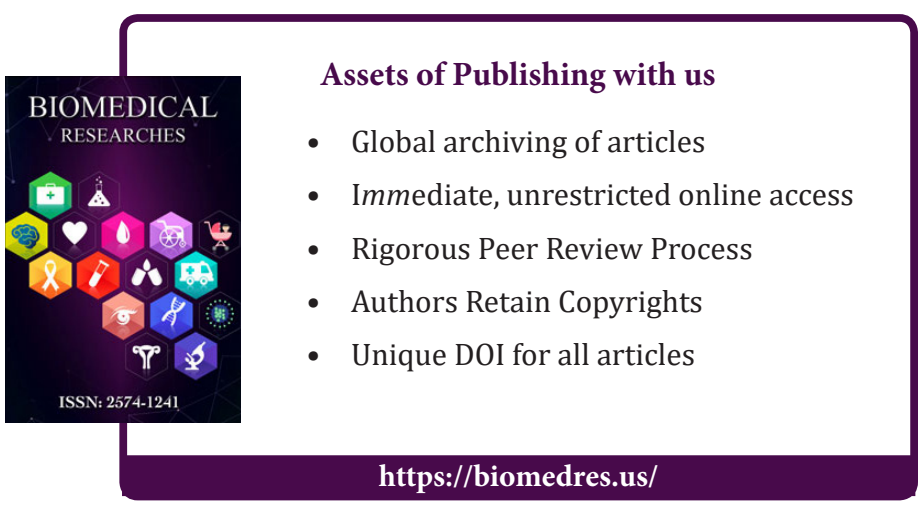

